\title{
Acceptor removal - Displacement damage effects involving the shallow acceptor doping of p-type silicon devices
}

\author{
Michael Moll ${ }^{* \dagger}$ \\ CERN, Geneva, Switzerland \\ E-mail: michael.moll@cern.ch
}

New sensor technologies are under development to cope with the ever increasing requirements for high energy physics (HEP) detectors. For the High-Luminosity LHC (HL-LHC) and the Future Circular Collider (FCC) higher radiation hardness, improved timing performance and lower cost large area detector technologies are essential. Recent developments of p-type silicon planar sensors, sensors with intrinsic gain for timing applications and monolithic sensors are examples for next generation detector technologies. A commonality of these new sensors is the use of boron doped p-type silicon, while present HEP sensors are essentially based on n-type silicon. Consequently, radiation effects in p-type silicon have been less studied in the detector community, but are now of high relevance. In this paper, we review radiation effects in p-type silicon devices with a focus on acceptor removal effects.

In p-type silicon exposure to radiation causes an apparent deactivation of the dopant. This socalled acceptor removal effect is for example evidenced by an initial decrease of depletion voltage in p-type sensors with rising particle fluence, while after exposure to higher fluences the device behavior is dominated by other radiation induced effects. Low Gain Avalanche Detectors (LGADs) are the baseline technology for future solid-state timing layers at the HL-LHC. Their good timing performance is based on the intrinsic amplification of the signal by impact ionization in a highly doped p-type gain layer. Radiation effects reduce the active boron concentration in the gain layer and lead to a reduction in field strength and consequently a loss of intrinsic signal amplification, thus limiting the radiation hardness of this technology. Monolithic detectors, especially High Voltage CMOS, are another technology affected by acceptor removal, as the bulk silicon used for the sensor is typically of p-type. We review the impact of radiation damage on the depleted volume as function of the initial boron concentration and the corresponding change in signal charge and device performance. The origin of the radiation induced deactivation of the boron acceptor is found in the creation of defects that contain boron, that is consequently no longer acting as shallow dopant. Defect spectroscopy techniques like Thermally Stimulated Currents (TSC) and Deep Level Transient Spectroscopy (DLTS) allow detecting these levels, help to understand the defect formation kinetics on the microscopic level and open the door to defect engineering approaches to mitigate the acceptor removal effect. Latest results of the CERN RD50 collaboration in this research field are reviewed.

The 28th International Workshop on Vertex Detectors - Vertex2019

13-18 October, 2019

Lopud, Croatia 


\section{Introduction}

Radiation induced displacement damage effects in silicon devices for High Energy Physics (HEP) experiments at the Large Hadron Collider (LHC) at CERN are a major concern for the present detector operation and future upgrades [1]. Within the frame of the High Luminosity LHC upgrade and anticipating the Future Circular Collider, a wide range of p-type silicon-based sensors are developed and studied. Prominent recent developments are Low Gain Avalanche Detectors (LGADs) for precision timing [2,3] and High Voltage CMOS sensors for inner tracking detectors [4]. All p-type devices are facing a particular radiation induced problem: The transformation of electrically active shallow acceptors into defect complexes that are no longer having these properties, the so-called acceptor removal effect. On the macroscopic level, the effect can be observed as a radiation induced change of depletion voltage $\left(V_{d e p}\right)$, respectively changed effective doping concentration of the space charge region $\left(N_{e f f}\right)$, a loss or increase of active sensor volume or a loss of signal gain. On the microscopic level, boron, which is mostly used in p-type silicon production, is removed from the substitutional lattice site $\left(B_{S}\right)$ and bound into defect complexes like $\left(B_{i} O_{i}\right.$ Boron interstitial-Oxygen interstitial or $B_{s} S i_{i}$ - Boron substitutional-Silicon interstitial) which no longer exhibit shallow acceptor properties. Understanding this boron removal effect is an essential step towards defect engineering and other mitigation approaches.

Most of the experimental data available are based on the measurement of a depletion depth $W_{\text {depl }}$ in a $n^{+}-p$ junction device, which is translated under the assumption of an abrupt junction and a homogeneous space charge into an effective space charge density $N_{\text {eff }}$

$$
W_{\text {depl }} \approx \sqrt{\frac{2 \cdot \varepsilon}{q_{0}\left|N_{e f f}\right|} \cdot V_{\text {bias }}}
$$

where $q_{0}$ is the elementary charge and $\varepsilon$ the permittivity of silicon. While this approach will be used in the following, it has to be mentioned that it has several shortcomings as it does not take into account the partly very complex electric field distribution in irradiated silicon sensors, inhomogeneous space charge distributions or the non-idealistic conditions for Capacitance-Voltage (CV) measurements on heavily irradiated sensors. The fluence dependence of the effective space charge under the assumption that the initial doping concentration is entirely given by an acceptor concentration $N_{A, 0}$ is generally parameterized as (see e.g. [1])

$$
N_{e f f}\left(\phi_{e q}\right)=N_{A, 0} \exp \left(-c_{A} \phi_{e q}\right)+g \phi_{e q}
$$

with an acceptor removal coefficient $c_{A}$ and an introduction rate $g$ covering all other radiation induce space charge generation mechanisms. While this parameterization can be fitted well to the data, the removal coefficient becomes dependent on the initial acceptor concentration $c_{A}\left(N_{A, 0}\right)$, and the exponential function itself is not reflecting the expectations from defect kinetics considerations (see section 6).

In the following, experimental data on the acceptor removal coefficient as obtained on CMOS sensors (Sec.2), LGADs (Sec.3) and silicon diodes (Sec.4) will be reviewed and recent defect

\footnotetext{
*Speaker.

${ }^{\dagger}$ On behalf of the RD50 collaboration.
} 
characterization studies on p-type silicon will be presented (Sec.5). The experimental data are discussed (Sec.6) and summarized in the conclusions (Sec.7).

\section{Acceptor removal in CMOS sensors}

In depleted CMOS pixel sensors the readout electronics, or part of it, are already embedded in the chip. Typically, a deep n-well embeds the transistors and acts as an electrode implant in a p-type substrate. A voltage is applied between the n-well and the sensor bulk leading to the growth of a depleted volume that serves to collect charges generated by particles or photons by charge drift. The depleted volume, respectively the depletion depth, for a given voltage depends on the resistivity of the substrate and can, for example, be probed with the edge-TCT [5] or TPA-TCT [6] methods. An example for such a measurement is given in Fig.1. The response of the sensor to a pulsed laser beam directed to the edge of the sensor at different depths (arrows in Fig.1(a) and y-position in Fig.1(b)) is measured. From the signal charge as function of depth and voltage (Fig.1(b)) the depletion depth $W_{d e p l}$ as function of applied voltage can be estimated (Fig.1(c)). A fit to the data with $W=W_{0}+$ $W_{\text {depl }}$ allows then to extract the effective doping concentration $N_{\text {eff }}$ (see Eq.1.1), respectively the acceptor concentration $N_{A}$ and the resistivity $\rho$. Under the assumption that this relationship holds for irradiated sensors, the evolution of the effective doping concentration as function of fluence $N_{e f f}\left(\phi_{e q}\right)$ can be analysed and eventually fitted to an expression like Eq.1.2 to obtain the acceptor removal coefficient $c_{A}$. This is shown for three different sensor types in Fig.2 (left). The resulting $c_{A}$ values as function of the initial doping concentration $\left|N_{e f f, 0}\right| \approx N_{A, 0}$ for neutron irradiated CMOS sensors are given in Fig.2 (right) with further data from literature and will be discussed in Sec. 6.

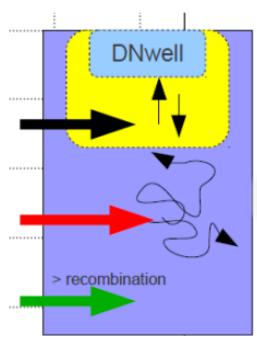

(a)

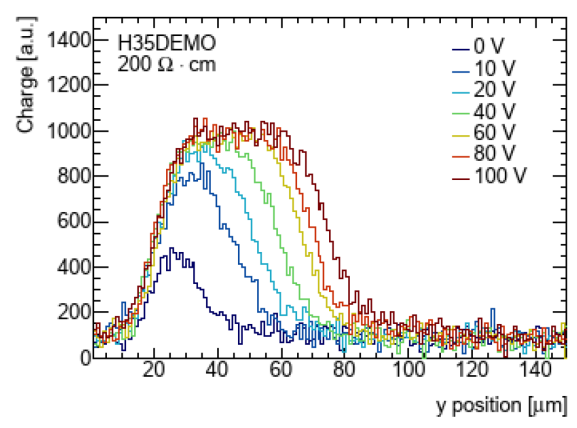

(b)

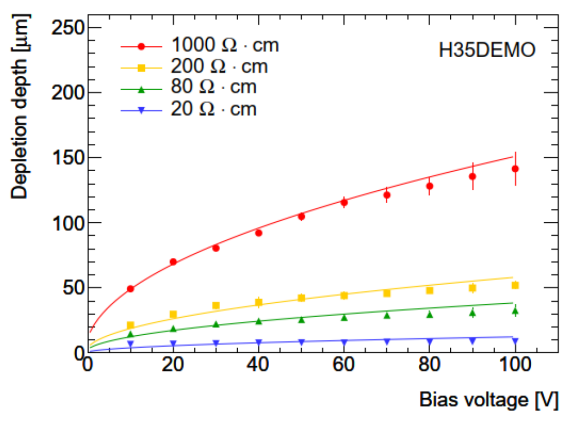

(c)

Figure 1: Use of edge-TCT measurements to extract the effective doping concentration. The arrows in (a) indicate illumination at different depth which correspond to the y-position in (b). The resulting depletion width is given as function of voltage in (c) (figures (b) and (c) from [7]).

\section{Acceptor removal in LGAD sensors}

Low Gain Avalanche Detectors (LGADs) exhibit a thin and highly p-type doped region (gain layer) which is generating a strong electric field with sufficient field strength to produce impact ionization for traversing electrons [2]. Fig.3 (left) gives a sketch of the corresponding $n^{++}-p^{+}-p-p^{++}$ device structure. The gain layer $\left(p^{+}\right)$is produced by a boron implantation that penetrates deeper 

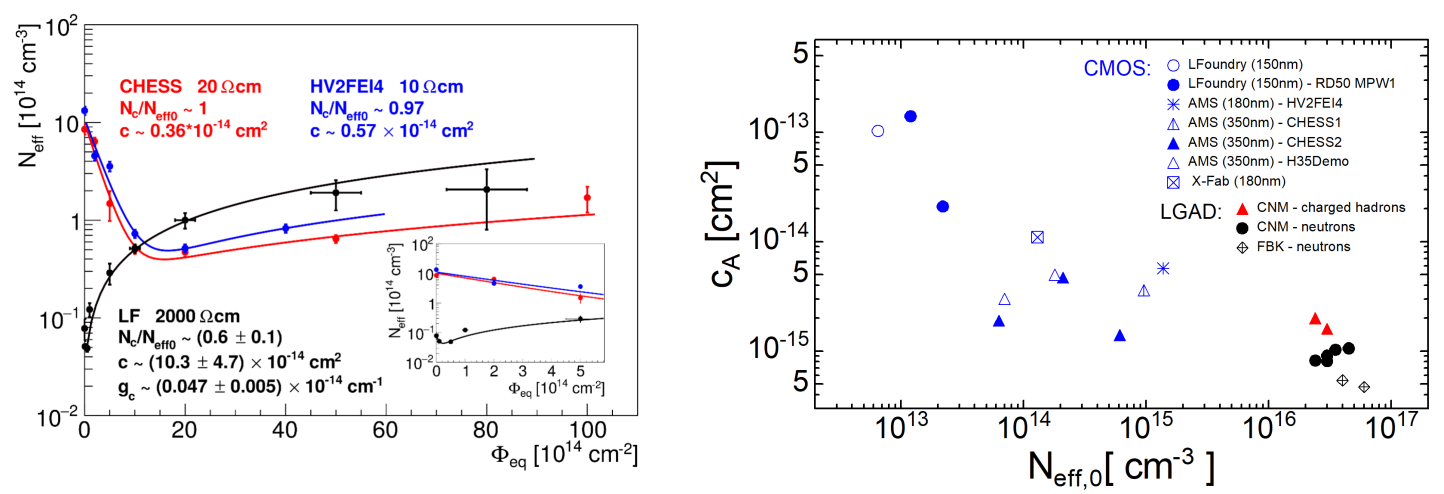

Figure 2: (left) Effective doping concentration dependence on neutron fluence for three different CMOS sensor types. The inset figure shows a zoom of the low fluence range. The curves are fits to the data resulting in the acceptor removal coefficient $c_{A}$ given in the right-hand figure (taken from [11]). (right) Acceptor removal coefficient $c_{A}$ as function of initial doping concentration $N_{e f f, 0}$ for CMOS sensors and LGADs. The values for CMOS sensors were obtained from edge-TCT measurements on neutron irradiated devices. The legend gives the CMOS foundry, the technology node and the name of the used chip design. For LGAD sensors the removal coefficient was extracted from measurements of the gain layer depletion voltage as function of fluence (see section 3). Data taken from [8, 9, 10, 11, 12, 13] for CMOS sensors and from $[14,16]$ for LGADs.

into the silicon bulk than the shallow phosphorus implant $\left(n^{++}\right)$that constitutes the cathode of the device. In this configuration with a boron implant dose of the order of $2 \times 10^{13} \mathrm{~cm}^{-2}$ the gain layer reaches a peak space charge density in the order of some $10^{16} \mathrm{~cm}^{-3}[2,14]$. The resulting intrinsic gain of the device (i.e. the signal increase wrt. an identical device without gain layer measured under identical conditions) is in the order of 10. The main obstacle for the operation in high energy physics experiments is the radiation induced degradation of the gain. An example of signal degradation is given in Fig.3 (right). With increasing voltage, first the gain layer and then the bulk of the LGAD is depleted, leading to a stable gain of about 2 in the given example for the non-irradiated device over a wide voltage range. With increasing radiation fluence the signal
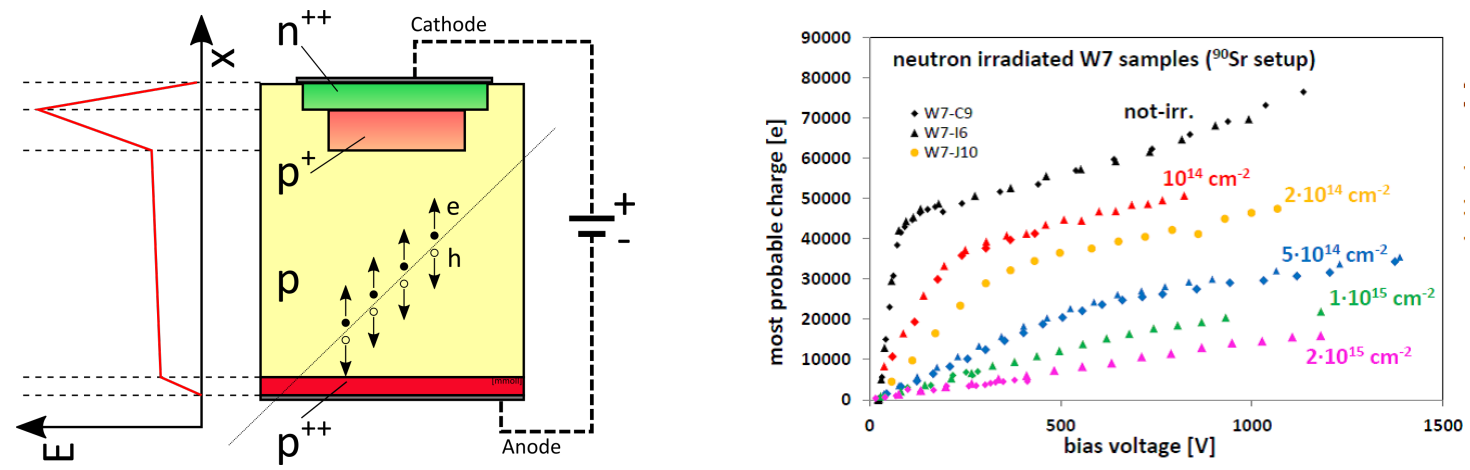

Figure 3: (left) Schematic view of the LGAD concept. (right) Dependence of the most probable charge $\left({ }^{90} \mathrm{Sr}\right.$ source) on bias voltage at different equivalent fluences for neutron irradiated LGAD. Data obtained at a temperature of $-10{ }^{\circ} \mathrm{C}$ after an annealing for $80 \mathrm{~min}$ at $60{ }^{\circ} \mathrm{C}$ prior to the measurements (from [14]). 

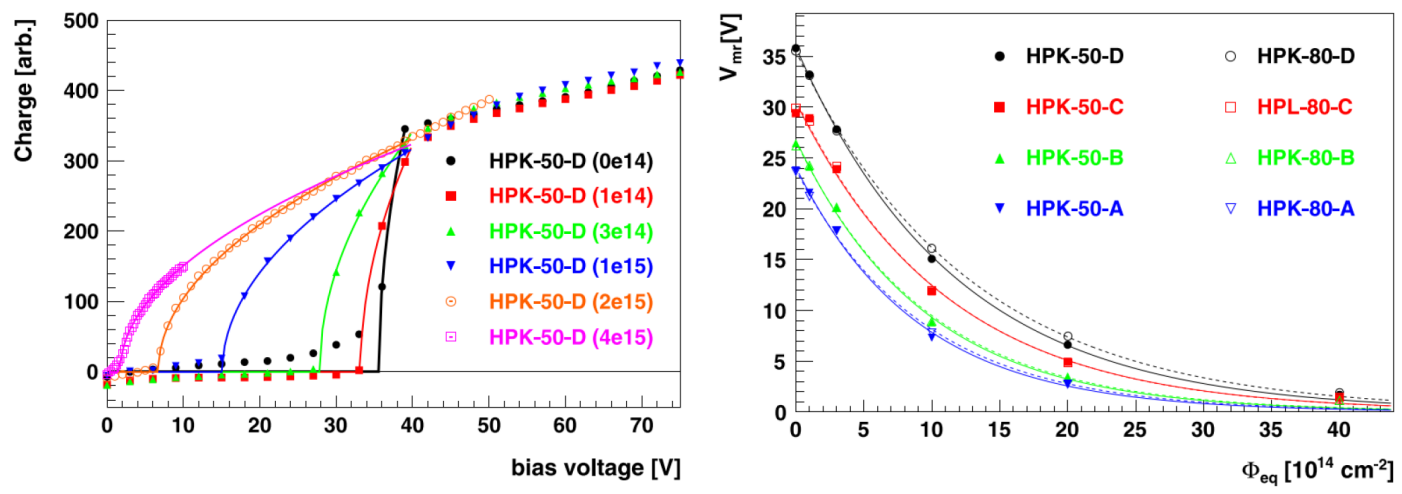

Figure 4: Transient Current Technique (TCT) measurements of LGAD sensors irradiated with different neutron fluences (front side illumination, $660 \mathrm{~nm}, 400 \mathrm{ps}, 500 \mathrm{~Hz}$ ). (left) The integrated signal current (20 ns) against applied voltage rises sharply when the gain layer is depleted $\left(V=V_{m r}\right)$. With increasing fluence the voltage $V_{m r}$ required to deplete the gain layer decreases. (right) The voltage $V_{m r}$ decreases exponentially with increasing fluence. The values for the sensor type labeled HPK-50-D have been extracted from the data shown in the left-hand figure (plots taken from [15]).

and the gain are reduced. The gain finally completely disappears, depending on the used LGAD device technology and particle used for irradiation, after a fluence of some $10^{15} \mathrm{~cm}^{-2}$ [14]. The origin of the gain degradation is found in a reduction of space charge density in the gain layer and reflected in the voltage required to deplete the gain layer. This is depicted in Fig.4 (left). The signal obtained under front side (cathode) illumination of LGADs with short laser pulses is plotted against the applied reverse voltage. A sharp rise in signal is observed when the gain layer is depleted $\left(V=V_{m r}\right)$. With increasing radiation fluence $\phi_{e q}$ a lower voltage $V_{m r}$ is required to obtain a signal, respectively to deplete the gain layer. This is also shown in Fig.4 (right), where an exponential function $V_{m r}=V_{m r, 0} \cdot \exp \left(-c \phi_{e q}\right)$ is fitted to the data. Under the assumption that the voltage $V_{m r}$ is a direct expression of the space charge within the gain layer, the obtained parameter $c$ corresponds to the acceptor removal coefficient $c_{A}$. The change of the depletion voltage of the gain layer $V_{m r}$ as function of irradiation fluence has been characterized by signal measurements employing TCT techniques [15] or radioactive sources [14] or by analysing Capacitance-Voltage (CV) measurements [16]. The corresponding $c_{A}$ values are given in Fig.2. While the extraction of the $c_{A}$ value is evident from the fit to the data, the value for the initial acceptor density $N_{A, 0}$ is afflicted with uncertainties due to the fact that the gain layer is a concentration profile of partly unknown width and shape. For LGADs defect engineering and gain layer profiling approaches have shown to impact on the removal rate [16]. These data are not included in Fig.2 (right). Especially a moderate increase of the carbon concentration within the gain layer has shown to have a beneficial effect of reducing the removal rate and thus increasing the radiation hardness of LGADs [16]. The LGAD data in Fig. 2 confirm the trend of reduced removal parameter with increasing initial acceptor concentration. Furthermore, charged hadron irradiations show a stronger acceptor removal than neutron irradiations for same equivalent fluences. This will be further discussed in sections 5 and 6 . 

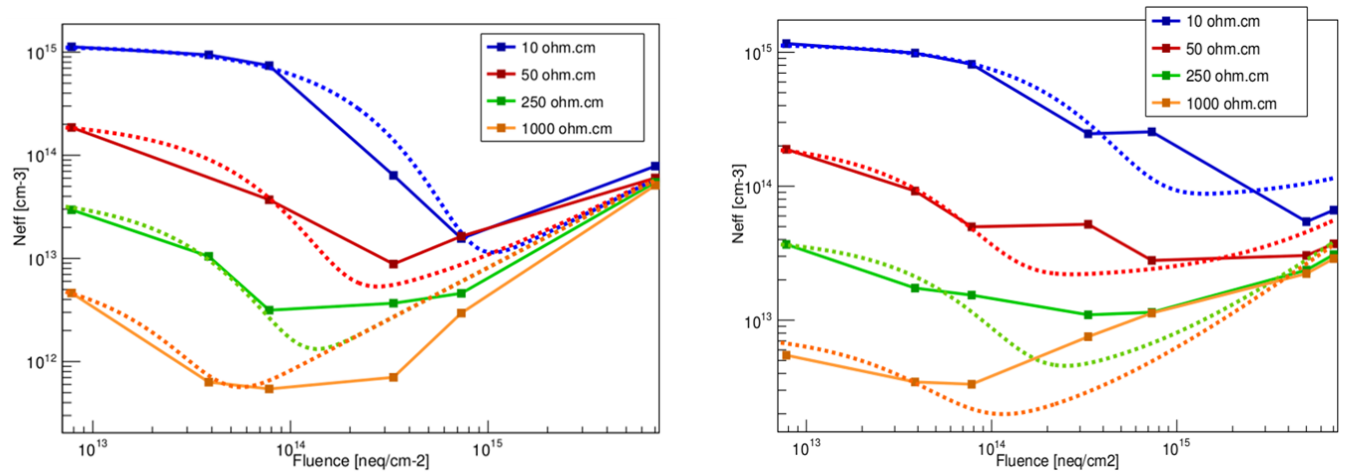

Figure 5: Effective doping concentration for p-type diodes made from epitaxial silicon with various resistivities as function of fluence $\left(\phi_{e q}\right)$. The data have been extracted from $\mathrm{CV}$ measurements after irradiation with $23 \mathrm{GeV}$ protons (left) and reactor neutrons (right). The dashed lines are fits to the data employing Eq.1.2 (plots with preliminary data taken from [17]).

\section{Acceptor removal in p-type wafers and sensors}

In a recent experiment a series of $n^{+}-p$ diodes have been produced on epitaxial silicon wafers with different resistivities ranging from 10 to $1000 \Omega \mathrm{cm}$ [17, 23]. Fig.5 shows the effective space charge density as function of fluence as extracted from $\mathrm{CV}$ measurements under the assumptions discussed for the validity of Eq.1.1 in Sec.1. It is evident that the effective doping concentration for the proton irradiated sensors changes faster than the one for the identical neutron irradiated devices. An interesting fact is that the $\mathrm{CV}$ curves and according depletion voltages of the samples irradiated to the highest fluence of $7 \times 10^{15} \mathrm{~cm}^{-2}$ are very similar. The initial doping concentration seems to have no (for proton irradiated samples) or only little (for neutron irradiated samples) impact on the data obtained at very high fluences. An observation resembling the donor removal process on high resistivity n-type silicon $[18,19]$. The dashed line in Fig.5 correspond to fits to the data according to Eq.1.2. The corresponding values for the acceptor removal coefficient are given in Fig. 6 together with other data extracted from literature on irradiated p-type diodes and wafers $[20,21,22,17]$. The trend of reduced acceptor removal coefficients with increasing initial acceptor content is clearly visible, while the data show an overall strong scattering pointing towards further hidden parameters like e.g. the impurity content that will require further work for understanding. Furthermore, a significant enhancement of the removal rate for proton irradiated samples against neutron irradiated samples of same type is observed, despite the fact that in both experiments the fluence has been normalized to the NIEL. This is a clear indication that point defects play a major role in the removal process (see e.g. [1]).

\section{Defect kinetics and defect characterization}

In order to understand the microscopic origin of the above described acceptor removal effects dedicated defect characterization campaigns on irradiated p-type silicon devices employing Deep Level Transient Spectroscopy (DLTS), Thermally Stimulated Current (TSC) and other techniques have been initiated within the RD50 collaboration [23, 24, 25]. A schematic understanding of 


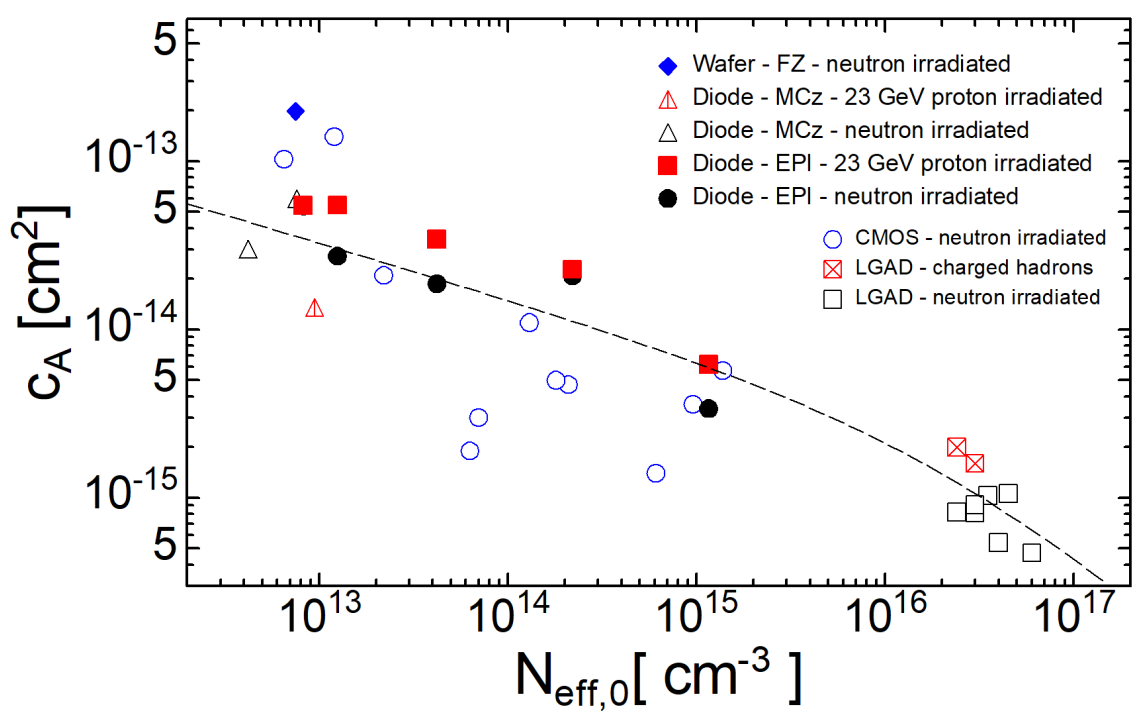

Figure 6: Acceptor removal coefficient $c_{A}$ as function of initial doping concentration $N_{e f f, 0}$ for p-type silicon sensors and wafers as deduced from literature. A more detailed legend for the CMOS and LGAD sensors is given in Fig.2. The line represents a parameterization with parameters given in [16].
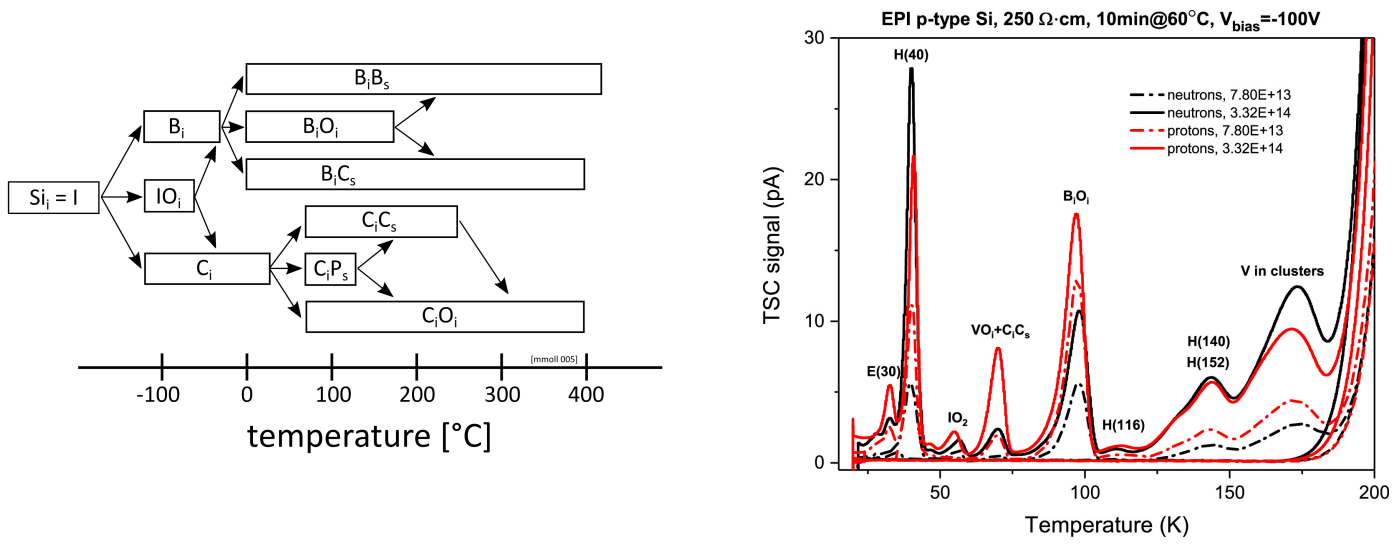

Figure 7: Left: Silicon self-interstitial defect reactions with impurities. The temperature scale shows the stability of the defects under quiescent conditions (adapted from [26]). Right: Thermally Stimulated Current (TSC) spectra obtained on p-type EPI pad diodes $(250 \Omega \mathrm{cm})$ after $23 \mathrm{GeV}$ proton (red) and neutron (black) irradiation with $7.8 \times 10^{13} \mathrm{n}_{\mathrm{eq}} \mathrm{cm}^{-2}$ (dashed lines) or $3.3 \times 10^{14} \mathrm{n}_{\mathrm{eq}} \mathrm{cm}^{-2}$ (solid lines) and annealing of $10 \mathrm{~min}$ at $60{ }^{\circ} \mathrm{C}$ (from [23]).

the boron deactivation process is given in Fig.7 (left). Under irradiation with highly energetic particles, defect clusters, interstitials $\left(S i_{i}\right.$ or $\left.I\right)$ and vacancies $(V)$ are formed in the silicon crystal lattice (see e.g. references in [1]). Both, vacancies and interstitials are mobile at temperatures close to room temperature and react with impurities, as idealized in the quasichemical reaction scheme in Fig.7 (left). In particular the interstitials do react with boron $\left(B_{s}\right)$, oxygen $\left(O_{i}\right)$ and carbon $\left(C_{s}\right)$. In a so-called Watkins replacement mechanism the interstitial can regain a lattice site in the crystal by reacting with a boron or carbon atom positioned on a lattice site. In this reaction (e.g. $I+B_{s} \rightarrow B_{i}$ ) 
the boron or carbon atoms become themselves interstitials, migrate through the lattice and can further react with other impurities producing other defect complexes (e.g. $B_{i}+O_{i} \rightarrow B_{i} O_{i}$ ). As the $I O_{i}$ complex is not stable at room temperature and in standard silicon the oxygen content is much higher than the carbon and boron content $([O] \gg[C],[B])$, the final states are expected to be $B_{i} O_{i}$ and $C_{i} O_{i}$.

An electron trap level at $E_{C}-0.23 \mathrm{eV}$ has been identified with the $B_{i} O_{i}$ defect [26], while recent works attributed potentially the $B_{s} I$ defect to this level [27]. Here, we will follow the more widely accepted $B_{i} O_{i}$ assignment. Independent of its assignment to a defect, the level has been identified as a donor level in the upper half of the bandgap and therefore provides positive space charge in the depletion region. Due to its large capture cross section for electrons it can be easily detected by DLTS and TSC after charge injection by forward biasing of the diode. Fig.7 (right) gives an example of TSC measurements on proton and neutron irradiated diodes produced on a $250 \Omega \mathrm{cm}$ boron doped epitaxial layer (see [23]). Samples irradiated with $23 \mathrm{GeV}$ protons show a higher $B_{i} O_{i}$ concentration as samples irradiated with reactor neutrons to the same $1-\mathrm{MeV}$ neutron equivalent fluence. This is expected due to the higher fraction of point defects in the proton irradiation (see [1] for related literature) and consistent with the fact that protons produce a stronger acceptor removal effect as compared to neutrons for the same neutron equivalent fluence.

The $C_{i} O_{i}$ defect is a hole trap with a donor level at $E_{V}+0.36 \mathrm{eV}$ and is therefore neutral in the space charge region [26]. Due to its small electron capture cross section it can not only be detected by standard DLTS in p-type silicon but also by charge injection methods in n-type silicon. The level exhibits however a very strong filling temperature dependence that does not allow to measure it in TSC measurements with low temperature injection [28]. This is the reason why it is not visible in the TSC measurement given in Fig.7 (right).

The work of the RD50 collaboration on the determination of absolute $B_{i} O_{i}$ and $C_{i} O_{i}$ concentrations in p-type silicon after various types of particle irradiation and material with different boron content has started. First preliminary measured introduction rates [23, 29] for the $B_{i} O_{i}$ concentration in form of a generation rate $g_{B i O i}=\left[B_{i} O_{i}\right] / \phi_{e q}$ are given in Fig.8 (right) and will be discussed in the following section.

\section{Discussion}

The acceptor removal effect in the LGAD, CMOS sensors and diodes in sections 2,3,4 was parameterized with an exponential function including an acceptor removal coefficient $c_{A}$. For small fluences this formulation can be approximated by

$$
N_{A}\left(\phi_{e q}\right)=N_{A, 0} \exp \left(-c_{A} \phi_{e q}\right) \approx N_{A, 0}-c_{A} N_{A, 0} \phi_{e q}=N_{A, 0}-g_{B} \phi_{e q}
$$

where $g_{B}=c_{A} \times N_{A, 0}$ is introduced as intial acceptor removal rate. Values for $g_{B}$ as function of the initial acceptor concentration $N_{A, 0}$ and corresponding to the data given in Fig.6 are shown in Fig. 8 (left). The representation gives a direct measure of the number of deactivated acceptors per unit volume and fluence. It demonstrates that the removal rate in absolute numbers is much higher for low resisitivity (i.e. high initial boron concentration) material. For silicon with an initial boron concentration of $N_{A, 0}=10^{13} \mathrm{~cm}^{-3}(\approx 1 \mathrm{k} \Omega \mathrm{cm})$ a removal rate of $\approx 0.3 \mathrm{~cm}^{-1}$ and for a material with $N_{A, 0}=10^{16} \mathrm{~cm}^{-3}(\approx 1 \Omega \mathrm{cm})$ a removal rate of $\approx 20 \mathrm{~cm}^{-1}$ is observed. According to the 

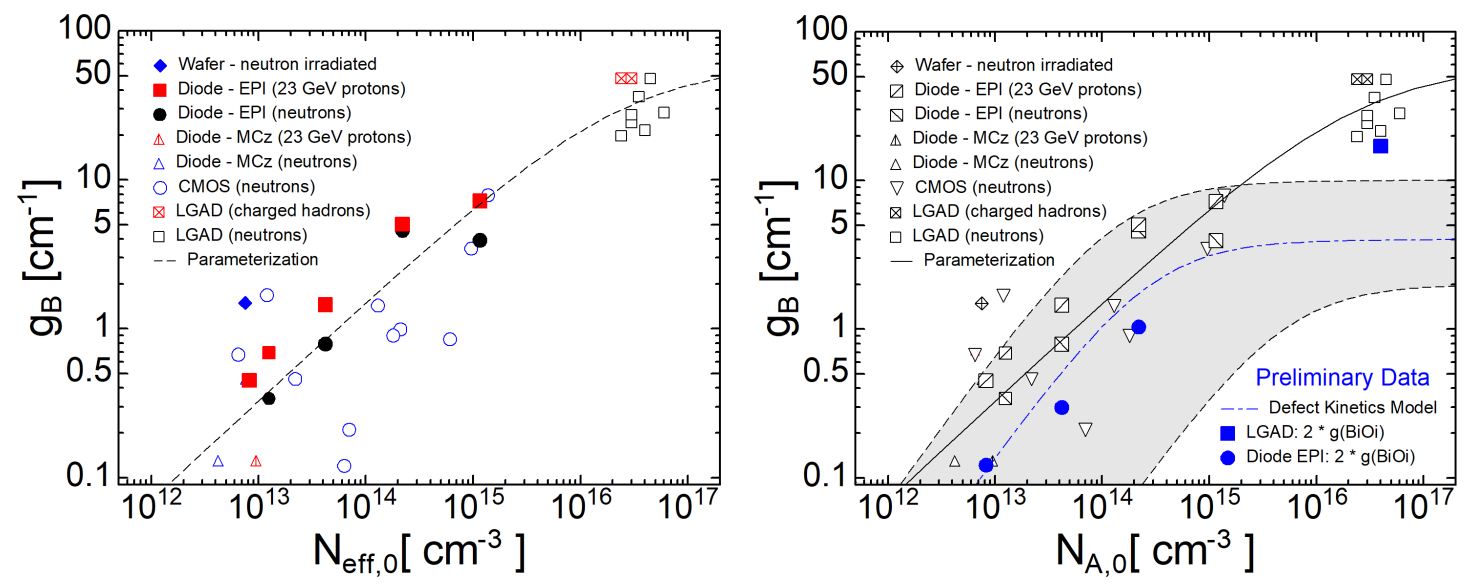

Figure 8: (left) Acceptor removal rates $g_{B}$ as function of initial doping concentration $N_{e f f, 0}$ for p-type silicon sensors and wafers as deduced from literature. The line represents a parameterization with parameters given in [16]. (right) Preliminary $B_{i} O_{i}$ introduction rates and a first principle defect kinetics model are added to the left-hand plot. Note that the $B_{i} O_{i}$ introduction rates and the model data are scaled with a factor of 2 to come up for the assumption that the $B_{i} O_{i}$ defect is positively charged (see text for more details).

parameterization given in [16] the removal rate then saturates around $60 \mathrm{~cm}^{-1}$ at very high boron content. From these observations, and based on the defect kinetics understanding given in Sec.5, we could interpret the data as follows: (a) About $60 \mathrm{~cm}^{-1}$ interstitials are produced by the primary particle interactions that then can react with impurities in the silicon. In the low resistivity silicon almost all interstitials will react with boron and lead to its deactivation, in high resistivity silicon most of the interstitials are captured at other impurities or are gettered at other sinks and only a small fraction of interstitials reacts with boron. (b) Another possibility is that in low resistivity silicon more interstitials are generated than in high resistivity material due to the higher concentration of charge carriers during irradiation.

These hypotheses can be evaluated against the defect kinetics model and the first experimental defect concentration data given in Sec.5: Under the assumption that the only possible defect reactions are the ones shown in Fig.7, almost all silicon interstitials (I) should finally end up in these two defects. Their concentration ratio $\left[C_{i} O_{i}\right] /\left[B_{i} O_{i}\right]$ should therefore reflect the ratio of $\left(k_{I C} / k_{I B}\right) \cdot\left[C_{S}\right] /\left[B_{S}\right]$ in the material, where the ratio of the capture radii of interstitials at boron $\left(k_{I B}\right)$ and at carbon $\left(k_{I C}\right)$ has been determined to be $k_{I B} / k_{I C} \approx 7$ [25]. The absolute boron removal rate $g_{B}$ is thus given by the generation rate $g_{B i O i}$ of the $B_{i} O_{i}$ defect which can be estimated to be

$$
g_{B} \approx g_{B_{i} O_{i}} \approx g_{I} \times\left(1+\frac{k_{I C}\left[C_{S}\right]}{k_{I B}\left[B_{S}\right]}\right)^{-1}
$$

Here the generation rate of interstitials $g_{I}$ represents the number of interstitials that escape the primary damage region and migrate throught the silicon lattice eventually reacting with impurities. Defect concentration measurements performed with DLTS and FTIR on high resistivity silicon sensors have shown that the interstitial related point defect generation rate is about $1-3 \mathrm{~cm}^{-1}[28$, 30]. Assuming $g_{I}=2 \mathrm{~cm}^{-1}$, a carbon concentration of $[C]=2 \times 10^{15} \mathrm{~cm}^{-3}$ and a scaling factor of 2 with respect to Eq.6.2 results in the dashed line given in Fig.8 (right). The factor 2 was introduced to come up for the understanding that in the removal process one shallow acceptor is 
removed (the $B_{s}$ ) and one charged deep donor is formed (the $B_{i} O_{i}$ ). The shaded area indicates the results of the model for the range $g_{I}=1-3 \mathrm{~cm}^{-1}$ and $[C]=1-5 \times 10^{15} \mathrm{~cm}^{-3}$, where an increase of the carbon concentration leads to a reduction in the boron removal rate. In general the measured data for the epitaxial diodes do not disagree with the basic defect kinetics model, while the first defect data obtained for LGADs as well as the experimental data obtained for the gain loss in LGADs cannot be explained by the simple defect formation model and the used input parameters. The LGAD acceptor removal $\left(N_{A, 0} \approx 5 \times 10^{16} \mathrm{~cm}^{-3}\right)$ and the given first defect measurement on LGADs could be explained by an interstitial introduction rate $g_{I}$ in the order of several $10 \mathrm{~cm}^{-1}$. This, however, calls for the question why the measured interstitial related defect concentrations in higher resistivity material is so much lower or, if the generation rate of the interstitials is identical in different resistivity material, where the interstitials have been gettered in the high resistivity material to reduce the rate of visible interstitial related defects to $\approx 2 \mathrm{~cm}^{-1}$. There is therefore no clear conclusion on the modelling of acceptor removal. Defect characterization campaigns on irradiated p-type silicon sensors are presently under way and should soon give a clearer picture on the physical processes underlying the acceptor removal effect.

\section{Conclusions}

The radiation induced de-activation of shallow acceptors (usually the boron doping) leads to a series of effects in p-type silicon devices operated in high radiation environments. The RD50 collaboration has studied LGAD, CMOS sensors and in particular p-type silicon detectors and diodes to characterize this so-called acceptor removal effect. Other radiation damage mechanisms not involving the shallow doping are also impacting on the devices and prohibit a clear view of the underlying microscopic mechanism or lead to mis-interpretation of data. The formation of the $B_{i} O_{i}$ defect has tentatively been identified as the main mechanism for the acceptor removal effect but further work is needed to consolidate this hypothesis. While there is a parameterization of the macroscopic removal effects, a fully quantitative understanding on the microscopic defect formation level is still lacking.

\section{References}

[1] M. Moll, "Displacement Damage in Silicon Detectors for High Energy Physics," IEEE Transactions on Nuclear Science, vol. 65, no. 8, pp. 1561-1582, Aug. 2018. doi: 10.1109/TNS.2018.2819506

[2] G. Pellegrini et al., Technology developments and first measurements of Low Gain Avalanche Detectors (LGAD) for high energy physics applications, NIMA 765 (2014) 12-16.

[3] H. Sadrozinski et al., 4D tracking with ultra-fast silicon detectors, Rep. Prog. Phys 81 (2018) 026101 (34pp).

[4] M. Garcia-Sciveres and N. Wermes, A review of advances in pixel detectors for experiments with high rate and radiation, Rep. Prog. Phys. 81 (2018) 066101 (43pp)

[5] G. Kramberger et al. Investigation of Irradiated Silicon Detectors by Edge-TCT, IEEE TNS 57, No.4 (2010), 2294. 
[6] M. Fernandez Garcia et al., On the determination of the substrate effective doping concentration of irradiated HV-CMOS sensors using an edge-TCT technique based on the Two-Photon-Absorption process, 2017 JINST 12 C01038.

[7] J. Anders et al., Charge collection characterisation with the Transient Current Technique of the ams H35DEMO CMOS detector after proton irradiation, JINST, P10004, 2018.

[8] A. Affolder et al., Charge collection studies in irradiated HV-CMOS particle detectors, JINST, P04007, 2016.

[9] E. Cavallaro et al., Studies of irradiated AMS H35 CMOS detectors for the ATLAS tracker upgrade, JINST, P10020, 2017.

[10] B. Hiti et al., Charge collection properties in an irradiated pixel sensor built in a thick-film HV-SOI process, JINST, P10020, 2017.

[11] I. Mandić et al., Neutron irradiation test of depleted CMOS pixel detector prototypes, JINST, P02021, 2017.

[12] B. Hiti et al., Charge collection in irradiated HV-CMOS detectors, NIMA 924, 214-218, 2019.

[13] I. Mandić et al., E-TCT measurements with passive CMOS pixel detectors on RD50-MPW1 chips from LFoundry, RD50 Workshop, CERN, November 2018.

[14] G. Kramberger et al., Radiation effects in Low Gain Avalanche Detectors after hadron irradiations, 2015 JINST 10 P07006.

[15] G. Kramberger et al., Radiation hardness of thin Low Gain Avalanche Detectors, NIMA 891 (2018) 68-77.

[16] M. Ferrero et al., Radiation resistant LGAD design, NIMA 919 (2019) 16-26.

[17] P.Almeida et al., Characterization of acceptor removal in silicon pad diodes irradiated by protons and neutrons, $32^{\text {nd }}$ RD50 Workshop, 4-6 June 2018, Hamburg.

[18] G.Lindström et al., Radiation hard silicon detectors - developments by the RD48 (ROSE) collaboration, NIMA 466 (2001) 308-326.

[19] M.Moll et al., Investigation of the improved radiation hardness of silicon detectors with high oxygen concentration, NIMA 439 (2000) 282-292.

[20] R.Wunstorf et al., Investigation of donor and acceptor removal and long term annealing in silicon with different boron/phosphorus ratios, NIMA 377 (1996) 228-233.

[21] V. Cindro et al., Radiation damage in p-type silicon irradiated with neutrons and protons, NIMA 599 (2009) 60-65.

[22] K. Kaska, $\mathrm{PhD}$ thesis, Characterization of radiation damage in detectors made from different silicon materials, Technische Universit'at Wien, 2014.

[23] Y.Gurimskaya et al., Radiation damage in p-type EPI silicon pad diodes irradiated with protons and neutrons, NIMA, in press. 2019. https://doi.org/10.1016/j.nima.2019.05.062

[24] L.F. Makarenko et al., Effect of electron injection on defect reactions in irradiated silicon containing boron, carbon, and oxygen, Journal of Applied Physics 123, 161576 (2018).

[25] L.F. Makarenko et al., Formation of a Bistable Interstitial Complex in Irradiated p-Type Silicon, Physica Status Solidi A 2019, 216, 1900354. 
[26] L.C. Kimerling et al., Interstitial defect reactions in silicon, Materials Science Forum Vols. 38-41 (1989) pp 141-150.

[27] K. Lauer et al., Discussion of $A_{S i}$-Si $i_{i}$-defect model in frame of experimental results on P line in indium doped silicon, Solid State Phenomena Vo.1. 242 (2016), 90-95.

[28] M. Moll, PhD thesis, Radiation Damage in Silicon Particle Detectors - microscopic defects and macroscopic properties -, Hamburg University, 1999.

[29] Ioana Pintilie, NIMP Bucharest, Romania and Yana Gurimskaya, CERN, Switzerland, private communication.

[30] G. Davies et al., Radiation damage in silicon exposed to high-energy protons, Physical Review B 73, 165202 (2006). 